\title{
A Highly Sensitive and Wide-Range Resonant Magnetic Micro-Sensor Based on A Buckled Micro- Beam
}

Nouha ALCHEIKH

King Abdullah University of Science and Technology

Sofiane Mbarek

King Abdullah University of Science and Technology

Mohammad Younis ( $D$ Mohammad.Younis@kaust.edu.sa)

King Abdullah University of Science and Technology

\section{Research Article}

Keywords: Lorentz-force, micro-sensor, electrothermally

Posted Date: November 25th, 2020

DOI: https://doi.org/10.21203/rs.3.rs-110938/v1

License: (c) (i) This work is licensed under a Creative Commons Attribution 4.0 International License.

Read Full License 


\section{Abstract}

We experimentally demonstrate a miniature highly sensitive wide-range resonant magnetic Lorentz-force micro-sensor. The concept is demonstrated based on the detection of the resonance frequency of an inplane electrothermally heated straight resonator operated near the buckling point. The frequency shift is measured with optical sensing and the device is operating at atmospheric pressure. The magnetometer demonstrates a sensitivity (S) of 33.9/T, which is very high compared to the state of the art. In addition, the micro-sensor shows a good linearity in wide range and low power consumption around $0.2 \mathrm{~mW}$. The above performances make the proposed micro-sensor promising for various low-cost magnetic applications.

\section{Introduction}

In recent years, microelectromechanical systems (MEMS) have increasingly attracted the attention of researchers for various applications, such as filtering ${ }^{1}$, signal processing ${ }^{9}$, energy harvesting ${ }^{10}$, and environmental sensors including magnetic, pressure ${ }^{2-4}$, accelerometer ${ }^{5}$, temperature ${ }^{6}$, flow ${ }^{7}$, and gas ${ }^{8}$. Magnetic field micro-sensors have been explored for various potential applications, such as magnetocardiography (MCG), magnetoencephalography (MEG), biomedical, inertial navigation systems, electronic compasses, telecommunications, and non-destructive testing ${ }^{11-15}$. These magnetic microsensors are mainly based on Lorentz force transduction. In addition to high sensitivity and low power consumption, Lorentz force magnetic MEMS sensors have important advantages, including low fabrication cost, high resolution, and compact size $\mathrm{s}^{14,16-19}$.

The intensive demand of MEMS structures has led to magnetic sensors based on Lorentz force MEMS resonators thanks to the high frequency shifting and resonance frequency tunability ${ }^{20-24}$. The frequency shift resonant magnetometer yields high accuracy, high outstanding stability, high sensitivity, low power consumption, and immunity to noise. Exposing heated structures to magnetic field creates uniform distributed forces perpendicular to the test structures affecting their stiffness. Hence, the field strength can be measured by detecting the resonance frequency shift of resonating structures.

Various techniques of magnetic field micro-resonators have been used, such as capacitive, piezoresistive, piezoelectric, and optical sensing techniques ${ }^{14,23,25-26}$. Despite the intrinsic losses from the device imperfections, optical sensing requires simple read-out electronic circuits, is more robust technique, and presents immunity to electromagnetic interference. In addition, this technique does not demand a vacuum package ${ }^{14}$.

The static and dynamic behaviours of electrothermally actuated in-plane micro-beams, have been extensively investigated ${ }^{27-32}$. In a previous work ${ }^{2}$, we exploited the buckling point of heated straight micro-beam to demonstrate highly sensitive and scalable pressure sensor. The concept is based on tracking the frequency shift of the buckled micro-beam upon changing the surrounding air pressure. Based on the same concept, we extended our study to demonstrate highly sensitive gas sensors ${ }^{8}$. The 
method is based on the simultaneous recording of the frequencies of the first and second modes while changing the gas concentration and type.

A small and low-cost magnetic sensor with high sensitivity in wide range, which is also simple in fabrication, operation, and sensing scheme, would be highly desirable. In this paper, we aim to realize a highly sensitive and wide range magnetic sensors based on electrothermally heated straight micro-beam operated near the buckling point. The method is based on measuring the frequency shift of the fundamental resonance frequency of micro-beam upon changing the magnetic field. Operating near the buckling point offers significant shifting in frequency, and thus high sensitivity in wide range of magnetic field.

\section{Background And Measurement Method}

The concept is illustrated in Fig. 1. First, the variation in the resonant frequency of the first mode is monitored under DC current $\left(\mathrm{I}_{\mathrm{Th}}\right)$. As shown in Fig. 1a, by increasing $\mathrm{I}_{\mathrm{Th}}$, the resonant frequency of the micro-beam decreases due to increase in its stiffness until the buckling point where the micro-beam's stiffness is almost zero. After buckling, an increase in the resonant frequency is observed due to the stretching mechanism, which increases the stiffness of the buckled micro-beam. Next, as shown in Fig. $1 \mathrm{~b}$, a low DC electrostatic voltage $\left(V_{D C}\right)$ induces a static deflection that leads to decrease in the dip of the first resonance frequency. Note here that the straight micro-resonator takes advantage to high frequency shifting by operating around the buckling bifurcation at which the micro-beam is very sensitive to a small stiffness change. By applying a magnetic field while operating around buckling point, it is possible to add a deflection which leads to an increase in the frequency shifting, Fig. 1c. Hence, the concept is based on tracking the fundamental natural frequency of the electrothermally buckled straight micro-beam upon exposing it to a wide range of magnetic field.

A schematic of the micro-sensor is shown in Fig 2a. To demonstrate the concept, we utilize a microresonator fabricated from a highly doped silicon device layer of silicon-on-insulator (SOI) wafer from MEMSCAP wafer. The micro-beam is of length $(L) 800 \mu \mathrm{m}$, width $(h) 2 \mu \mathrm{m}, 25 \mu \mathrm{m}$ depth $(b)$, and is separated with two adjacent electrodes of $8 \mu \mathrm{m}$ gap $(g)$. A schematic of the proposed Lorentz-force magnetic sensor is shown in Fig. $2 \mathrm{~b}$. The electrothermal voltage $\mathrm{V}_{\mathrm{Th}}$ is applied between the two anchors inducing a current $\mathrm{I}_{\mathrm{Th}}$ flowing through the micro-beam that generates heat. In addition, the straight beam is electrostatically actuated by a $D C$ voltage $V_{D C}$. As seen in Fig. $2 b$, with the presence of a magnetic field $\left(B_{Z}\right)$ and with a DC current $I_{T h}$, which flows through the micro-beam, Lorentz-force $\left(F_{Y}\right)$ is generated normal to the straight micro-beam in $\mathrm{Y}$-axis.

Schematic of the experimental setup used to test the micro-resonator is shown in Fig. 2c. The resonant frequency shifting is measured using a laser Doppler vibrometer from Polytec ${ }^{33}$ (MSA 500) as actuating the straight micro-beam with a white noise signal. The driving electrode of the resonator is electrically connected to a DC and AC harmonic voltage sources provided by the MSA. Also, the micro-beam is electrothermally actuated by a separate $\mathrm{DC}$ voltage source $\mathrm{V}_{\mathrm{Th}}$. To generate a DC magnetic field in z-axis 
$\left(B_{Z}\right)$, a Neodymium permanent magnet is positioned above the sensor. All experiments are conducted at pressure atmospheric.

\section{Results And Discussions}

Figure 3a shows the variation in the measured fundamental resonance frequency of the micro-beam, while changing the input current $\mathrm{I}_{\mathrm{Th}}$ and for 0 magnetic field $\left(\mathrm{B}_{\mathrm{Z}}=0 \mathrm{mT}\right)$. It can be observed that the resonance frequency decreases with the increase in $\mathrm{I}_{\mathrm{Th}}$ and reaches a minimum value around $0.27 \mathrm{~mA}$, which corresponds to the buckling point. By increasing $\mathrm{I}_{T h}$, the compressive axial load induced through the micro-beam increases via Joules heating, which also causes decrease in its stiffness. After buckling, a sharp increase in the resonant frequency is observed, which increases the stiffness of the buckled micro-beam. Note here that the buckling bifurcation is utilized since the micro-beam becomes very sensitive to any small stiffness change. A small electrostatic voltage $\left(V_{D C}=16 \mathrm{~V}\right)$ is applied between the lower driving electrode and micro-beam, which creates a small deflection in Y-axis, and thus causes decrease in the dip (above zero) of the first resonance frequency ${ }^{27}$. Note that the thermal time constant of the proposed magnetic sensor is around $176 \mu \mathrm{s}^{8}$. For faster operation, the micro-sensor needs to be placed in vacuum.

The variation in the resonance frequency, while changing $I_{T h}$ and for varying magnetic field strength $\left(B_{Z}\right)$, is shown in Fig. 3b. By increasing $B_{Z}$, the Lorentz-force $\left(F_{Y}\right)$ increases, which also causes increase in initial deflection, and thus increases the resonant frequency of the resonator. The results show that the resonance frequency dip is almost eliminated for high value of $B_{Z}(400 \mathrm{mT})$, which explains that the micro-beam experiences a high perturbed bifurcation due to the existence of magnetic field. In addition, we observe that the frequency responses can be measured with wide range of $B_{Z}$ strengths from $4 \mathrm{mT}$ to $400 \mathrm{mT}$ at atmospheric pressure. However, our proposed micro-sensor can detect a magnetic field lower than $4 \mathrm{mT}$.

Next, we discuss the sensitivity of the micro-sensor $S(1 / T)$, which is defined as the relative change in the resonant frequency $\left(\Delta f / f_{0}\right)$ over the variation of an input magnetic $\left(\partial B_{Z}\right)^{20}$. The frequency shift $(\Delta f)$ is defined as $\left(f-f_{0}\right)$, where $f_{0}$ and $f$, are respectively, the frequency of the micro-beam at $0 m T$ and during the measurement with $B_{Z}$. We first measured $\Delta f$ with $B_{Z}$ as fixing $I_{T h}$ at $0.14 \mathrm{~mA}$ (before buckling) and 0.27 $\mathrm{mA}$ (at the buckling point), respectively, Fig. 4a. For both values of $\mathrm{I}_{\mathrm{Th}}$, the results show two linear trends, which separate the magnetic strengths in two ranges; low $\left(B_{Z} \leq 8 \mathrm{mT}\right)$ and high range $\left(B_{Z} \geq 8 \mathrm{mT}\right)$. As shown, the frequency shift (resulting from the same field strength) is found higher at $\mathrm{I}_{\mathrm{Th}}=0.27 \mathrm{~mA}$ where the micro-beam stiffness reaches almost zero. Hence, operating the resonator near the buckling point maximizes the frequency shift.

As we mention above, the measured linear coefficient of the relative change in the resonant frequency as a function of increasing magnetic field can represent the sensitivity (S) of the micro-sensor. Figs. $4 \mathrm{~b}$ and $4 \mathrm{c}$ present $\Delta f / f_{0}$ measurements against $B_{Z}$ at a bias current of $0.14 \mathrm{~mA}$, away from the buckling point, for 
both low and high magnetic field ranges. As shown in Figs. $4(\mathrm{~b}, \mathrm{c})$, the sensitivity at low range is $8.46 / \mathrm{T}$, and for high range is $0.35 / \mathrm{T}$. It is observed that, through linear fitting, the micro-sensor shows high linearity for both ranges. We next plot the results for both ranges around the bucking point $(0.27 \mathrm{~mA})$, Figs. 4(d,e). The slopes of the plots yield a sensitivity of $33.9 / \mathrm{T}$ for the low range and $2.56 / \mathrm{T}$ for the high range. Hence, the device can sense with high sensitivity a wide range of magnetic field. These values are four and seven times larger than the values at $0.14 \mathrm{~mA}$. This confirms well with the concept that by operating at the buckling point, the proposed micro-sensor is very sensitive to any external force including from magnetic fields. The results indicate that the dependence of $S$ on $B_{Z}$ becomes more significant in low range compared to high ranges. The improved high sensitivity at low ranges encourages the efforts toward low cost magnetic sensors applications.

Next, we show the results of $S$ with $\mathrm{I}_{\mathrm{Th}}$ to have an understanding of the relationship between sensitivity and power consumption. Figs. $5(\mathrm{a}, \mathrm{b})$ plot the sensitivity versus $\mathrm{I}_{\mathrm{Th}}$ for the two ranges. The results show that $S$ increases as a cubic polynomial with $\mathrm{I}_{\mathrm{Th}}$. Increasing the current from $0.1 \mathrm{~mA}$ to $0.27 \mathrm{~mA}$ (before buckling point), the sensitivity can be further improved by $870 \%$ for the low range and $800 \%$ for the high range. This again confirms that the sensor sensitivity becomes high as operating around the buckling point. It also shows that the operating current point of the magnetic sensor can be tuned to achieve higher sensitivity.

One should mention that the proposed sensor might be promising for some applications, which require high sensitivity. In addition to having high sensitivity, low power consumption is also an important factor. At bucking point $(0.27 \mathrm{~mA})$, the sensor consumes power around $0.2 \mathrm{~mW}$ due to the electrothermal actuation. This value can be reduced to half by operating at $0.135 \mathrm{~mA}$ while there is significant reduction in S (81\%). Hence, with a straight micro-beam, high sensitivity is achieved even for a low input current. However, the power of the device can be improved by reducing the cooling effect between the micro-beam and the surrounding air (e.g., operating at low pressure using vacuumed package).

Note here that the current detection method using optical readout system (laser) does not suffer considerably from noise, electronic circuitry, and weight. In other sensing methods, where resonances are detected electrically, such as capacitive sensing, they suffer from parasitic capacitances and other sources of noise, which can have high impact on the resolution of measurements. Furthermore, the proposed sensing technique does not compensate the environmental temperature variation, which represents one limitation of the frequency based magnetic sensors compared to capacitive technique. Moreover, calibration experiments may be conducted to compensate the variation of ambient temperature.

Table 1 below shows comparison for the performance of the proposed micro-sensor to some recent works on Lorentz force resonant magnetic field micro-sensors. As listed in Table 1, the sensitivity of the device is significantly higher and with smaller dimensions compared with other previously reported magnetic sensors. Also, it is noted that the results show high sensitivity in wide range of magnetic field 
strengths. In addition, the proposed micro-sensor is useful to reduce the device power and its size, and thus the cost of the MEMS magnetometer.

Table 1. Summary of the performance of some of the recent MEMS magnetic field sensors based on Lorentz force.

\begin{tabular}{|c|c|c|c|c|c|}
\hline Reference & $\begin{array}{l}\text { Magnetic range } \\
(\mathrm{mT})\end{array}$ & $\begin{array}{l}\text { Power } \\
(\mathrm{mW})\end{array}$ & $\begin{array}{c}\text { Surface } \\
\left(\mathrm{mm}^{2}\right)\end{array}$ & $\begin{array}{l}\text { Sensitivity } \\
\left(m A^{-1} \cdot T^{-1}\right)\end{array}$ & $\begin{array}{l}\text { Sensing } \\
\text { method }\end{array}$ \\
\hline [Zhang et al. [20]] & $\leq 100$ & 40 & 0.48 & 33.9 ppm & Capacitive \\
\hline [Bahreyni et al. [34]] & {$[2.5-25]$} & $0.1-10$ & 0.27 & $332.3 \mathrm{ppm}$ & Capacitive \\
\hline $\begin{array}{l}\text { [Herrera-May et al. } \\
\text { [35]] }\end{array}$ & $\leq 7$ & 10 & 0.06 & 922.7 ppm & Piezoresistive \\
\hline [Laghi et al. [36]] & {$[-5$ to 5$]$} & 10 & 0.308 & 2800000 ppm & Capacitive \\
\hline This work & $\leq 8$ & 0.2 & 0.0144 & $\begin{array}{l}125000000 \\
\mathrm{ppm}\end{array}$ & Optical \\
\hline This work & [8-400] & 0.2 & 0.0144 & 9500000 ppm & Optical \\
\hline
\end{tabular}

\section{Conclusions}

In this paper, we demonstrated experimentally a highly sensitive in-plane Lorentz-force magnetic microsensor by exploiting the buckling point bifurcation of a straight beam. The concept is based on the measured resonance frequency shift of the first mode under different magnetic strengths. The microsensor demonstrates high sensitivity compared to reported magnetic sensors. The main advantages of the proposed sensor are the simplicity of fabrication, low-cost, excellent linearity, low power consumption, scalability, and wide range measurements. The high performances above motivate in-depth studies for the implementation of the proposed magnetic micro-sensor in low magnetic fields strengths around $\mu \mathrm{T}$. In conclusion, we demonstrated that a single electrothermal straight micro-resonator could be potentially used as a magnetic sensor by measuring the frequency shift near the buckling point. Future studies are planned for measurements in vacuum to reduce the power consumption and lower the minimal detectable magnetic fields.

\section{Declarations}

\section{ACKNOWLEDGEMENT}

We acknowledge financial support from King Abdullah University of Science and Technology. 
The authors declare no conflict of interest.

\section{AUTHOR CONTRIBUTIONS}

N.A performed the measurements and analyzed the data. S.B.M. performed the measurements. M.I.Y. supervised the project.

\section{References}

1. Hajjaj AZ, Hafiz MA, Younis MI. Mode coupling and nonlinear resonances of MEMS arch resonators for bandpass filters. Scientific reports. 2017;7:41820.

2. Hajjaj AZ, Alcheikh N, Hafiz MA, llyas S, Younis MI. A scalable pressure sensor based on an electrothermally and electrostatically operated resonator. Applied Physics Letters. 2017;111(22):223503.

3. Alcheikh N, Hajjaj AZ, Younis MI. Highly sensitive and wide-range resonant pressure sensor based on the veering phenomenon. Sensors and Actuators A: Physical. 2019;300:111652.

4. Najar F, Ghommem M, Abdelkefi A. A double-side electrically-actuated arch microbeam for pressure sensing applications. International Journal of Mechanical Sciences. 2020 :105624.

5. Arif M, Milind P, Zhao C, Guillermo S, Du Z, Philipp S, Xudong Z, Howe RT, Seshia AA. A vibrating beam MEMS accelerometer for gravity and seismic measurements. Scientific Reports, 2020;10(1): 10415.

6. Jaber N, llyas S, Shekhah O, Eddaoudi M, Younis MI. Multimode MEMS resonator for simultaneous sensing of vapor concentration and temperature. IEEE Sensors Journal. 2018;18(24):10145-53.

7. Yarali M, Khanna SK. Microfabrication of a variable range and multi-directionally sensitive thermal flow sensor. Sensors and Actuators A: Physical. 2014;220:159-67.

8. Hajjaj AZ, Jaber N, Alcheikh N, Younis MI. A Resonant Gas Sensor Based on Multimode Excitation of a Buckled Microbeam. IEEE Sensors Journal. 2019;20(4):1778-85.

9. Moser J, Güttinger J, Eichler A, Esplandiu MJ, Liu D, Dykman M, et al. Ultrasensitive force detection with a nanotube mechanical resonator. Nature Nanotechnology. 2013;8(7):493-6.

10. Shi Q, Wang T, Lee C. MEMS Based Broadband Piezoelectric Ultrasonic Energy Harvester (PUEH) for Enabling Self-Powered Implantable Biomedical Devices. Scientific Reports. 2016;6.

11. Dominguez-Nicolas SM, Juarez-Aguirre R, Herrera-May AL, Garcia-Ramirez P, Figueras E, Gutierrez-D EA, Tapia JA, Trejo A, Manjarrez E. Respiratory magnetogram detected with a MEMS device. International journal of medical sciences. 2013;10(11):1445-50.

12. Javor J, Stange A, Pollock C, Fuhr N, Bishop DJ. 100 pT/cm single-point MEMS magnetic gradiometer from a commercial accelerometer. Microsystems \& Nanoengineering. 2020;6(1):1-3.

13. Mykhaylyk O, Dudchenko N, Dudchenko A. Doxorubicin magnetic conjugate targeting upon intravenous injection into mice: High gradient magnetic field inhibits the clearance of nanoparticles from the blood. Journal of magnetism and magnetic materials. 2005 ;293(1):473-82. 
14. Herrera-May AL, Aguilera-Cortés LA, García-Ramírez PJ, Manjarrez E. Resonant magnetic field sensors based on MEMS technology. Sensors. 2009; (10):7785-813.

15. Díaz-Michelena M. Small magnetic sensors for space applications. Sensors. 2009; 9(4):2271-88.

16. Thompson MJ, Horsley DA. Parametrically amplified Z-axis Lorentz force magnetometer. Journal of microelectromechanical systems. 2011;20(3):702-10

17. Herrera-May AL, Soler-Balcazar JC, Vázquez-Leal H, Martínez-Castillo J, Vigueras-Zuñiga MO, Aguilera-Cortés LA. Recent advances of MEMS resonators for Lorentz force based magnetic field sensors: design, applications and challenges. Sensors. 2016;16(9):1359.

18. Sonmezoglu S, Horsley DA. Reducing offset and bias instability in Lorentz force magnetic sensors through bias chopping. Journal of Microelectromechanical Systems. 2016;26(1):169-78.

19. ME Sánchez-Chiva JM, Valle J, Fernández D, Madrenas J. A mixed-signal control system for Lorentzforce resonant MEMS magnetometers. IEEE Sensors Journal. 2019;19(17):7479-88.

20. Zhang W, Lee JE. Frequency-based magnetic field sensing using Lorentz force axial strain modulation in a double-ended tuning fork. Sensors and Actuators A: Physical. 201;211:145-52.

21. Chen F, Zhou W, Zou H, Kraft M, Li X. Dual-Resonator MEMS Magnetometer Based on Self-Clocking Sigma-Delta Modulation. IEEE Sensors Journal. 2019;20(3):1527-35.

22. Li M, Nitzan S, Horsley DA. Frequency-modulated Lorentz force magnetometer with enhanced sensitivity via mechanical amplification. IEEE Electron Device Letters. 2014;36(1):62-4.

23. Park B, Li M, Liyanage S, Shafai C. Lorentz force based resonant MEMS magnetic-field sensor with optical readout. Sensors and Actuators A: Physical. $2016 ; 241: 12-8$.

24. Park S, Al-Ghamdi MS, Khater ME, Abdel-Rahman E. A tunable MEMS magnetic sensor. Journal of Microelectromechanical Systems. 2016;26(1):255-6

25. Wu G, Xu D, Xiong B, Feng D, Wang Y. Resonant magnetic field sensor with capacitive driving and electromagnetic induction sensing. IEEE electron device letters. 2013;34(3):459-61.

26. Herrera-May AL, Lara-Castro M, López-Huerta F, Gkotsis P, Raskin JP, Figueras E. A MEMS-based magnetic field sensor with simple resonant structure and linear electrical response. Microelectronic Engineering. 2015;142:12-21.

27. Hajjaj AZ, Alcheikh N, Ramini A, Hafiz MAA, Younis MI. Highly Tunable Electrothermally and Electrostatically Actuated Resonators. Journal of Microelectromechanical Systems. 2016;PP(99):110.

28. Hajjaj AZ, Alcheikh N, Younis MI. The static and dynamic behavior of MEMS arch resonators near veering and the impact of initial shapes. International Journal of Non-Linear Mechanics. 2017 ;95:277-86.

29. Hajjaj AZ, Ramini A, Alcheikh N, Younis MI. Electrothermally tunable arch resonator. Journal of Microelectromechanical Systems. 2017;26(4):837-45.

30. Behera AR, Shaik H, Rao GM, Pratap R. Experimental Investigation of Dynamic Characteristics of Metal Coated Buckled Micro-Beams with Electrothermal Modulation of Residual Stress. In2018 4th 
IEEE International Conference on Emerging Electronics (ICEE) 2018 Dec 17 (pp. 1-4). IEEE.

31. Bakri-Kassem M, Dhaouadi R, Arabi M, Estahbanati SV, Abdel-Rahman E. Nonlinear dynamic modeling of a V-shaped metal based thermally driven MEMS actuator for RF switches. Journal of Micromechanics and Microengineering. 2018;28(5):054004.

32. Amor A, Fernandes A, Pouget J. Snap-through of elastic bistable beam under contactless magnetic actuation. International Journal of Non-Linear Mechanics. 2020;119:103358.

33. [Online].Polytech: http://www.polytec.com/us/.

34. Bahreyni B, Shafai C. A resonant micromachined magnetic field sensor. IEEE Sensors Journal. 2007 ;7(9):1326-34.

35. Herrera-May AL, García-Ramírez PJ, Aguilera-Cortés LA, Martínez-Castillo J, Sauceda-Carvajal A, García-González L, Figueras-Costa E. A resonant magnetic field microsensor with high quality factor at atmospheric pressure. Journal of Micromechanics and Microengineering. 2008;19(1):015016.

36. Laghi G, Dellea S, Longoni A, Minotti P, Tocchio A, Zerbini S, Langfelder G. Torsional MEMS magnetometer operated off-resonance for in-plane magnetic field detection. Sensors and Actuators A: Physical. 2015 Jun 15;229:218-26.

\section{Figures}



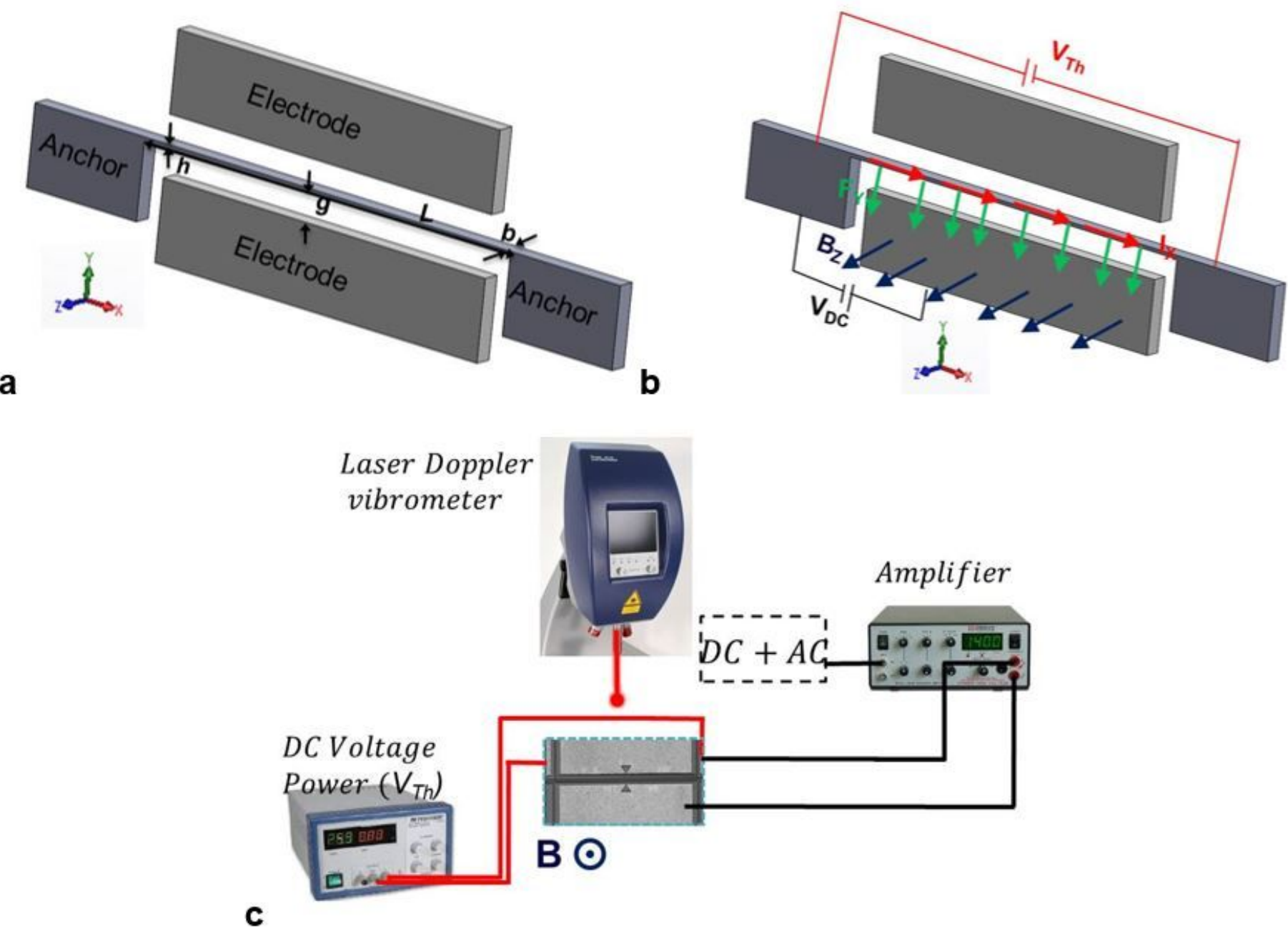

Figure 1

Schematics illustrating the principle of the proposed Lorentz-force magnetic sensor. (a) The first resonance frequency of the micro-beam for various DC current (ITh). (b) The first resonance frequency versus ITh showing the upward shift of buckling point due to a DC electrostatic polarization voltage (VDC). (c) Under magnetic field, the buckling point frequency dip experiences considerable upward frequency shift. 

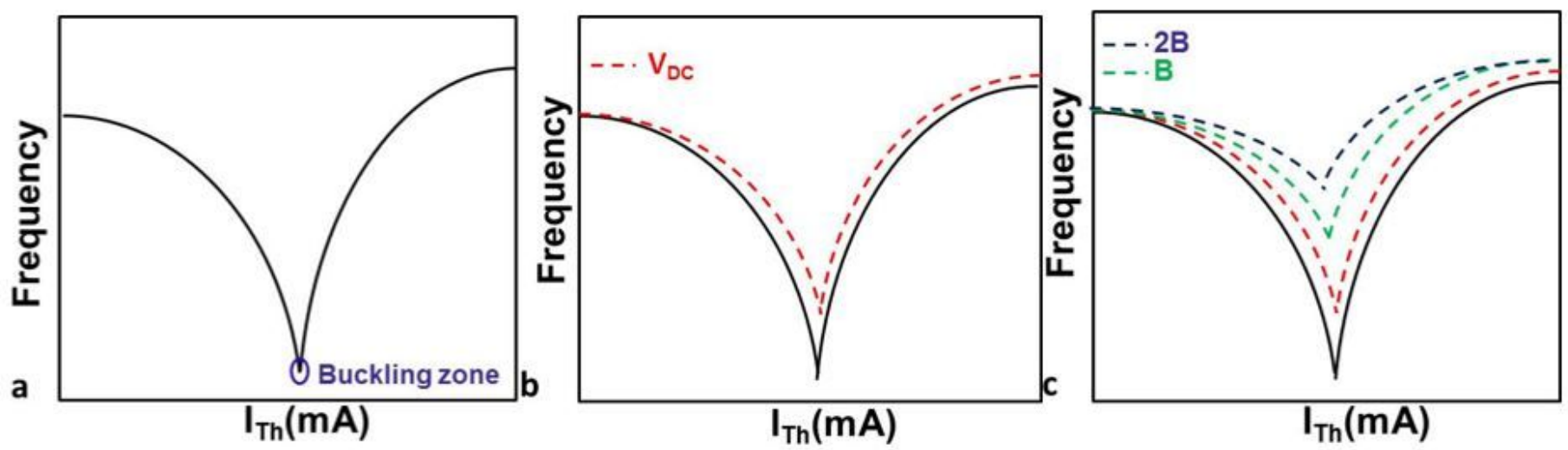

Figure 2

(a) A 3D schematic of the clamped-clamped straight micro-beam. (b) Schematic of the Lorentz-force magnetic sensor based on in-plane straight micro-beam electrothermally and electrostatically actuated. (c) Schematic of the experimental setup.
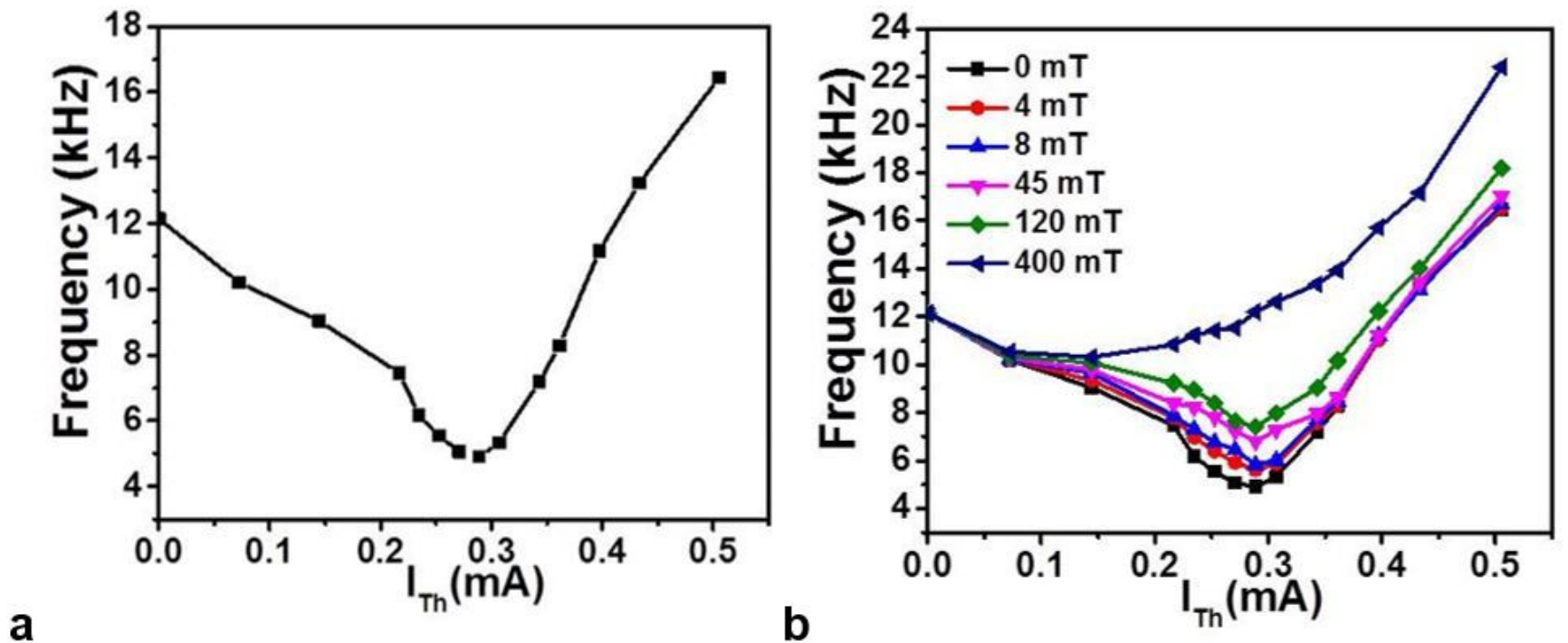

Figure 3

The first resonance frequency of the micro-beam under electrothermal actuation with $16 \mathrm{~V} \mathrm{DC}$ electrostatic polarization voltage (VDC) for (a) $B z=0 \mathrm{mT}$ and (b) for various values of $z$-axis magnetic fields. Note that the VDC bias perturbs the symmetric bifurcation at the buckling point (ITh=0.27 mA), which raises the resonance frequency at buckling above zero. 

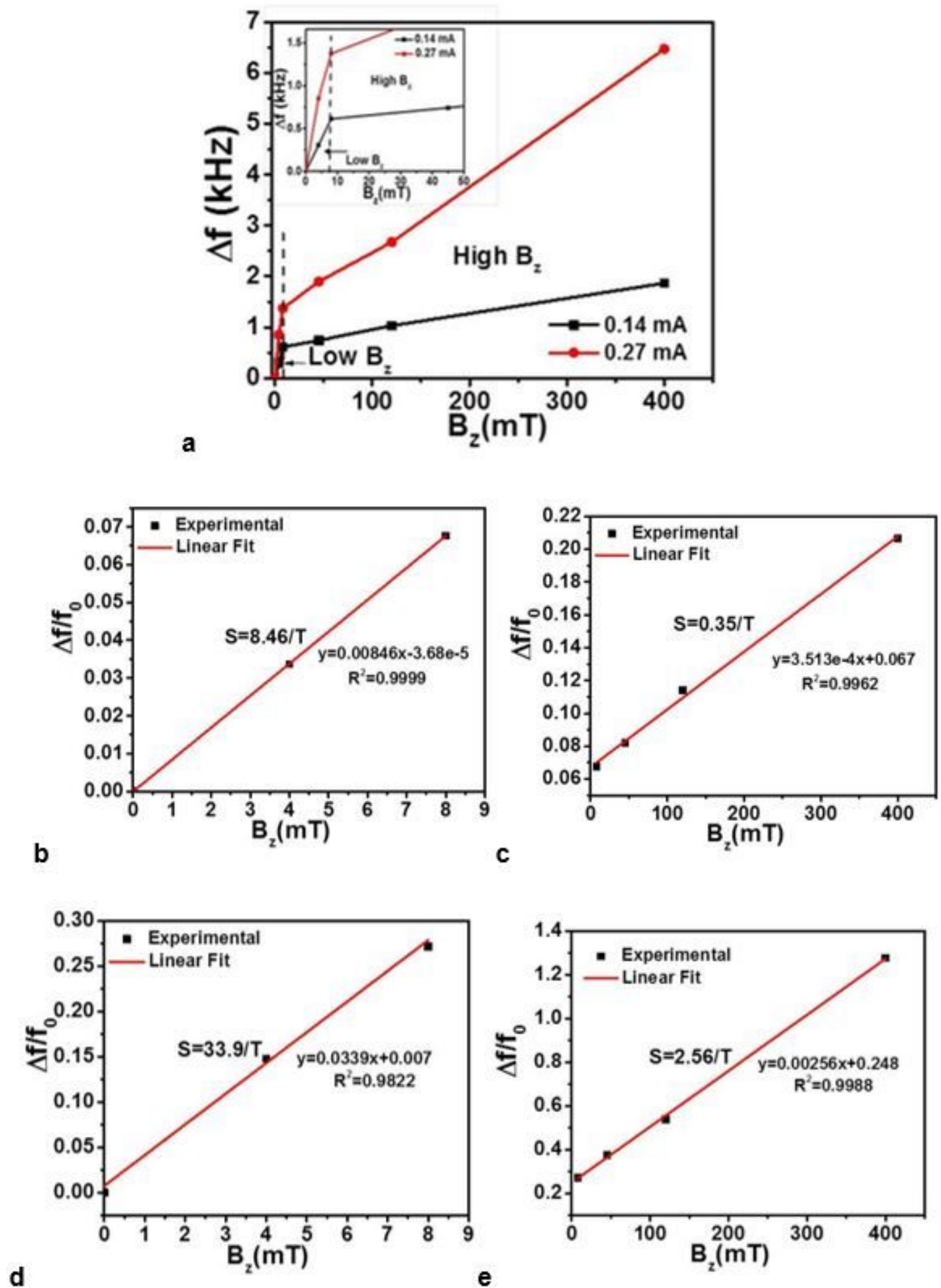

\section{Figure 4}

(a) Frequency shift versus input magnetic field (BZ) at ITh= $0.14 \mathrm{~mA}$ and $0.27 \mathrm{~mA}$. The vertical line splits the magnetic range strength as low $(B Z \leq 8 \mathrm{mT})$ and high $(B Z \geq 8 \mathrm{mT})$. The relative change in the resonant frequency $(\Delta f / f 0)$ versus $B Z$ at $(b, c) I T h=0.14 \mathrm{~mA}$ and $(\mathrm{d}, e) I T h=0.27 \mathrm{~mA}$; and for $(b, d)$ low and $(c, e)$ high magnetic ranges. f0 denotes the resonance frequency of the micro-beam at $0 \mathrm{mT}$. The sensitivity of the device (S) represents the linear coefficient of the variation of $\Delta \mathrm{f} / \mathrm{fO}$ as a function of $\mathrm{Bz}$. 

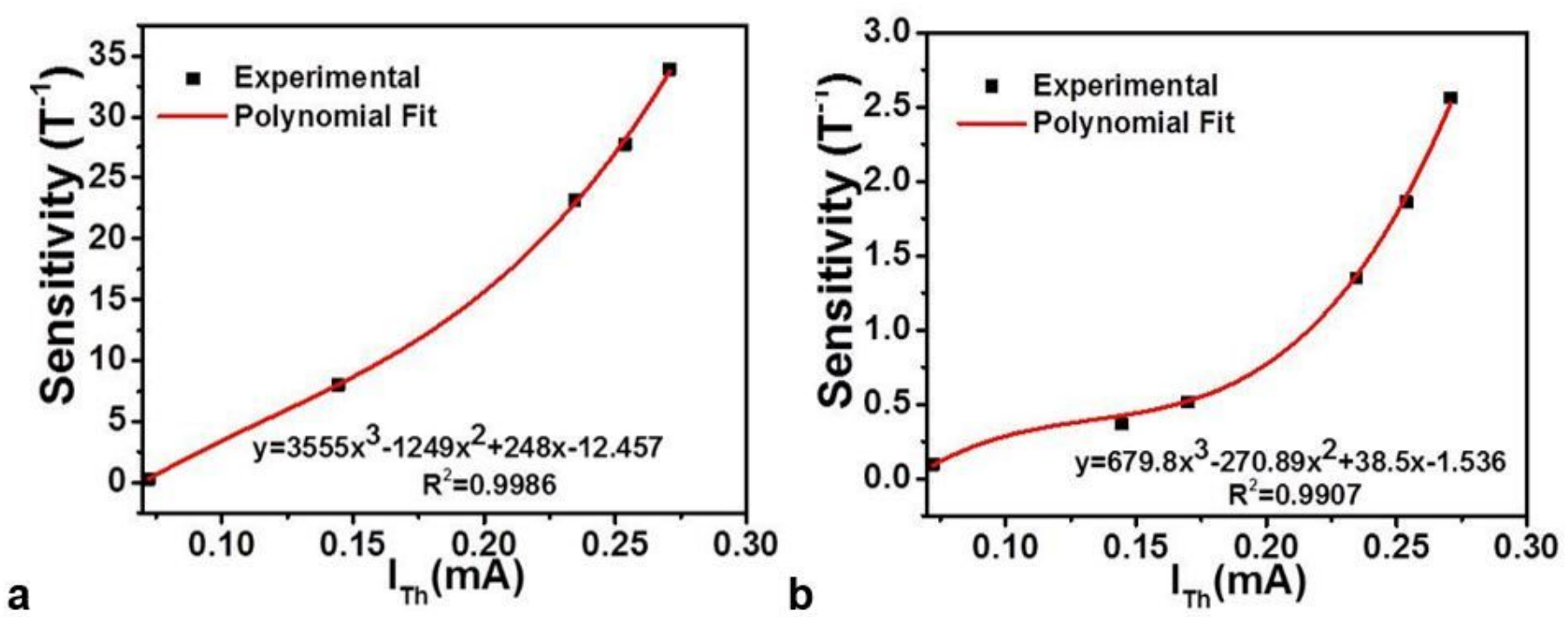

Figure 5

Sensitivity with increased bias current levels for (a) low and (b) high Bz strengths. 